\title{
RP105 ameliorates hypoxia/reoxygenation injury in cardiac microvascular endothelial cells by suppressing TLR4/MAPKs/NF-кB signaling
}

\author{
XIN GUO $^{1}$, HONG JIANG ${ }^{1}$, JING CHEN ${ }^{1}$, BO-FANG ZHANG ${ }^{1}$, QI HU ${ }^{1}$, \\ SHUO YANG ${ }^{1}$, JIAN YANG ${ }^{2}$ and JING ZHANG ${ }^{2}$ \\ ${ }^{1}$ Department of Cardiology, Renmin Hospital of Wuhan University, Cardiovascular Research Institute of Wuhan University, \\ Wuhan, Hubei 430060; ${ }^{2}$ Department of Cardiology, The First College of Clinical Medical Sciences, \\ China Three Gorges University, Yichang, Hubei 443000, P.R. China
}

Received May 21, 2016; Accepted December 5, 2017

DOI: $10.3892 / \mathrm{ijmm} .2018 .3621$

\begin{abstract}
The radioprotective $105 \mathrm{kDa}$ protein (RP105) has been implicated in the pathological process of multiple cardiovascular diseases through its functional and physical interactions with Toll-like receptor 4 (TLR4). However, the effects of RP105 on cardiac microvascular endothelial cells (CMECs) in response to hypoxia/reoxygenation $(\mathrm{H} / \mathrm{R})$ injury have not been extensively investigated. The aim of the present study was to elucidate the potential roles of RP105 in the protection of CMECs against H/R injury, and investigate the underlying mechanisms. CMECs isolated from Sprague-Dawley rats were transduced with adenoviral vectors encoding RP105 or green fluorescent protein (GFP). At $48 \mathrm{~h}$ post-transfection, CMECs were subjected to hypoxia for $4 \mathrm{~h}$ and reoxygenation for $2 \mathrm{~h}(\mathrm{H} / \mathrm{R})$ to simulate the in vivo ischemia/reperfusion model. The mRNA and protein levels of RP105 were detected by reverse transcription-quantitative polymerase chain reaction and western blot analysis, respectively. The effects of RP105 on CMEC proliferation, migration and apoptosis were measured by GFP-8, Transwell chamber and flow cytometry assays, respectively. The secretion of interleukin (IL)-6 and tumor necrosis factor (TNF)- $\alpha$ in the culture medium was measured by ELISA. Moreover, the expression level of TLR4, p38 mitogen-activated protein
\end{abstract}

Correspondence to: Dr Hong Jiang or Dr Jing Chen, Department of Cardiology, Renmin Hospital of Wuhan University, Cardiovascular Research Institute of Wuhan University, 238 Jiefang Road, Wuhan, Hubei 430060, P.R. China

E-mail: hong-jiang@whu.edu.cn

E-mail: gxqcl@sohu.com

Abbreviations: RP105, radioprotective $105 \mathrm{kDa}$ protein; CMECs, cardiac microvascular endothelial cells; H/R, hypoxia/reoxygenation; I/R, ischemia/reperfusion; TLRs, Toll-like receptors; MAPKs, mitogen activated protein kinases; TIR, Toll-IL-1 receptor

Key words: radioprotective $105 \mathrm{kDa}$ protein, cardiac microvascular endothelial cells, hypoxia/reoxygenation, Toll-like receptor 4 kinase (MAPK), extracellular-signal-regulated kinase 1/2, c-Jun N-terminal kinase, nuclear factor (NF)- $\mathrm{B} / \mathrm{p} 65$, IL-6, TNF- $\alpha$ and intercellular adhesion melecule-1 was evaluated by western blot analysis. The results demonstrated that RP105 was minimally expressed in CMECs subjected to H/R injury. Overexpression of RP105 via adenoviral vectors was able to significantly protect CMECs against H/R injury, as evidenced by the promotion of cell proliferation and migration, as well as the amelioration of inflammation and apoptosis. These beneficial effects were at least partly mediated through inhibition of TLR4/MAPKs/NF- $\kappa$ B signaling. Therefore, RP105 may be a promising candidate for prevention against CMECs-associated $\mathrm{H} / \mathrm{R}$ injury.

\section{Introduction}

Data from the World Health Organization (http://www.who. int/cardiovascular_diseases/en/) indicate that 17.5 million patients succumb to coronary vascular diseases annually, an estimated $31 \%$ of all deaths worldwide. A series of pharmacological and surgical therapies aimed at revascularization may rapidly reverse myocardial ischemia (1). However, the following ischemia/reperfusion (I/R) injury paradoxically alleviates the therapeutic benefits and results in further loss of cardiomyocytes and cardiac microvascular endothelial cells (CMECs) $(1,2)$. Great efforts have been made to investigate the pathogenetic processes underlying cardiomyocyte-associated I/R injury, while the critical roles of CMECs and the underlying mechanisms have attracted less attention (3). CMECs constitute approximately one-third of all cardiac cells, and act as first responders and central mediators in the regulation of $\mathrm{I} / \mathrm{R}$ injury (4-6). It is well known that CMEC injury precedes cardiomyocyte lesions under I/R conditions $(2,7)$. I/R treatment predisposes to dysfunction of CMECs, mainly through affecting their proliferation and migration, wherein inflammation- and apoptosis-dependent signaling pathways are strongly involved $(2,4,8)$. Moreover, there is close functional and physical crosstalk between cardiomyocytes and CMECs. For example, one single cardiomyocyte is surrounded by at least 3-4 myocardial capillaries (9); the apoptosis of 
CMECs and subsequent release of pro-inflammatory and pro-apoptotic mediators promote cardiomyocyte injury (10). These key characteristics of CMECs have prompted the search for interventional targets that may be directed at endogenous mechanisms, thereby reducing the side effects of I/R.

Toll-like receptor 4 (TLR4) is a common upstream sensor and acts as a pivotal initiator of multiple intracellular pathways (11). Previous investigations have highlighted the pleiotropic functions of TLR4 on regulating the malfunction of microvascular endothelial cells during hypoxia/reoxygenation $(\mathrm{H} / \mathrm{R})(10,12,13)$. Its detrimental effects are mainly due to the inflammation- and apoptosis-mediated cascade responses, as well as the impairment of proliferation and migration, which are in part modulated by the TLR4/nuclear factor (NF) $-\kappa B$ signaling pathway (12-14). Furthermore, previous studies have demonstrated that microvascular endothelial cells expressed mitogen-activated protein kinases (MAPKs), including p38 MAPK, c-Jun N-terminal kinase (JNK) and extracellular signal-regulated kinase 1/2 (ERK1/2), which serve as key downstream signaling molecules of TLR4 and are responsible for the initial inflammatory and apoptotic activation under ischemic conditions $(13,15)$. Of note, due to the abundant expression of TLRs and adhesion molecules, particularly TLR4 and intercellular adhesion melecule-1 (ICAM-1), CMECs act as 'sentinel' cells that may be more sensitive to H/R stimulation through sensing danger signals $(12,16)$. These findings may prove meaningful in the protection against $I / R$ injury by regulating TLR4, although the specific role of TLR4 and the more comprehensive molecular mechanisms that are involved in H/R-related CMEC injury require further elucidation.

The radioprotective $105 \mathrm{kDa}$ protein (RP105) serves as a TLR4 homologue, and is primarily expressed in mature stereotypical immunocytes $(17,18)$. Interestingly, RP105 may also be detectable in resident cardiac cell types, such as cardiomyocytes, vascular smooth muscle cells (VSMCs) and endothelial cells (18-20). Due to lack of the Toll/interleukin (IL)-1 receptor (TIR) domain, RP105 exerts its physiological actions mainly through its direct interactions with TLRs $(11,17)$. It is becoming increasingly clear that the inactivation of TLR4-triggered signaling pathways specifically regulated by RP105 was closely associated with the pathological processes of vascular remodeling, neointima formation and myocardial infarction $(21,22)$. However, whether RP105 exerts a direct effect on CMEC dysfunction under H/R conditions remains unknown. The aim of the present study was to determine whether the overexpression of RP105 attenuates H/R injury through limiting CMEC inflammation and apoptosis, and by enhancing their proliferation and migration abilities.

\section{Materials and methods}

CMEC isolation. CMECs were isolated from 60 male Sprague-Dawley (SD) rats, as previously described (23). Briefly, the hearts of anesthetized SD rats (weight, 80-100 g; age, 28 days) were quickly excised and rinsed with phosphate-buffered saline (PBS) containing heparin. After the atria, right ventricle and valves were removed, the left ventricle was immersed in $75 \%$ ethanol for $30 \mathrm{sec}$, and one-third of the outer free ventricular wall was discarded. The remaining tissues were washed by PBS, and incubated in $0.2 \%$ colla- genase for $10 \mathrm{~min}$ followed by $0.2 \%$ trypsin for $6 \mathrm{~min}$ at $37^{\circ} \mathrm{C}$. The dissociated cells were filtered using a $100-\mathrm{mm}$ mesh filter and centrifuged at $111 \mathrm{x} \mathrm{g}$ for $10 \mathrm{~min}$. The cells were re-suspended in Dulbecco's modified Eagle's medium (DMEM) containing 20\% fetal calf serum (FCS), $1 \%$ endothelial cell growth factor, $1 \%$ endothelial cell growth supplement, $0.5 \%$ penicillin/streptomycin and $0.12 \%$ heparin, and then seeded onto dishes. The primary CMECs were cultured in an incubator with $5 \% \mathrm{CO}_{2}$ at $37^{\circ} \mathrm{C}$. The identification of CMECs was based on the morphological characteristics and typical CD31 immunofluorescence assay, as previous depicted (2). All animal care and experimental procedures were approved by the Institutional Animal Care and Use Committee of Wuhan University, and conformed to the Guidelines for the Care and Use of Laboratory Animals published by the US National Institutes of Health.

Adenovirus transduction. Adenoviral vectors encoding RP105 (Ad-RP105) or control Ad-green fluorescent protein (GFP) was designed as previously demonstrated and provided by GeneChem (Shanghai, China) (24). Prior to adenoviral transfection, CMECs $\left(1 \times 10^{6} / \mathrm{ml}\right)$ were seeded into 6 -well plates until they reached $\sim 80 \%$ confluence. The cells were then incubated with Ad-RP105 or Ad-GFP at various multiplicities of infection (MOI) for $48 \mathrm{~h}$. The cytotoxic effects of adenovirus on CMECs were measured by $4 \%$ trypan blue dye, as reported previously (25). Fluorescent microscopy was used to observe GFP expression. The mRNA level of RP105 was further measured to evaluate the effectiveness of adenoviral transfection.

Experimental designations and $H / R$ model. Passage 2-3 CMECs were used for the following experiments. Following synchronization for $6 \mathrm{~h}$, the CMECs were rinsed with PBS and randomly designated into four groups: i) Control; ii) H/R; iii) Ad-GFP+H/R; and iv) Ad-RP105+H/R. At $48 \mathrm{~h}$ post-adenoviral transfection, the CMECs were subjected to $4 \mathrm{~h}$ of hypoxia followed by $2 \mathrm{~h}$ reoxygenation to simulate the in vivo I/R injury model, as previously described $(4,8)$. Hypoxia was achieved by exposing CMECs to $94 \% \mathrm{~N}_{2}-5 \% \mathrm{CO}_{2}-1 \% \mathrm{O}_{2}$ for $4 \mathrm{~h}$ at $37^{\circ} \mathrm{C}$ in Hanks' solution. Subsequently, the buffer was replaced by fresh culture medium and transferred into a normoxic incubator $\left(95 \%\right.$ air- $5 \% \mathrm{CO}_{2}$ ) for $2 \mathrm{~h}$ to initiate reoxygenation. The control group was maintained in a normoxic environment. All experiments were performed in triplicate and repeated three times.

Cell proliferation detection. Cell proliferation was detected by the Cell Counting Kit-8 (CCK-8) assay (Dojindo, Tokyo, Japan) according to the manufacturer's instructions (2). The CMECs were seeded onto 96-well plates at a density of $1 \times 10^{4}$ cells/well and subjected to $\mathrm{H} / \mathrm{R}$ injury as described above, until reaching $\sim 80 \%$ confluence. Subsequently, $10 \mu \mathrm{l} \mathrm{CCK}-8$ reagents were added into each well and incubated for $3 \mathrm{~h}$ in a $5 \% \mathrm{CO}_{2}$ incubator. The absorbance at $450 \mathrm{~nm}$ was measured using a microplate reader (Bio-Rad Laboratories, Hercules, CA, USA). Cell viability (\%) was expressed as the ratio of mean absorbance in the test wells to mean absorbance in the control well.

Cell migration measurement. Cell migration was measured using a 24-well Transwell chamber with an $8-\mu \mathrm{m}$ pore 
A
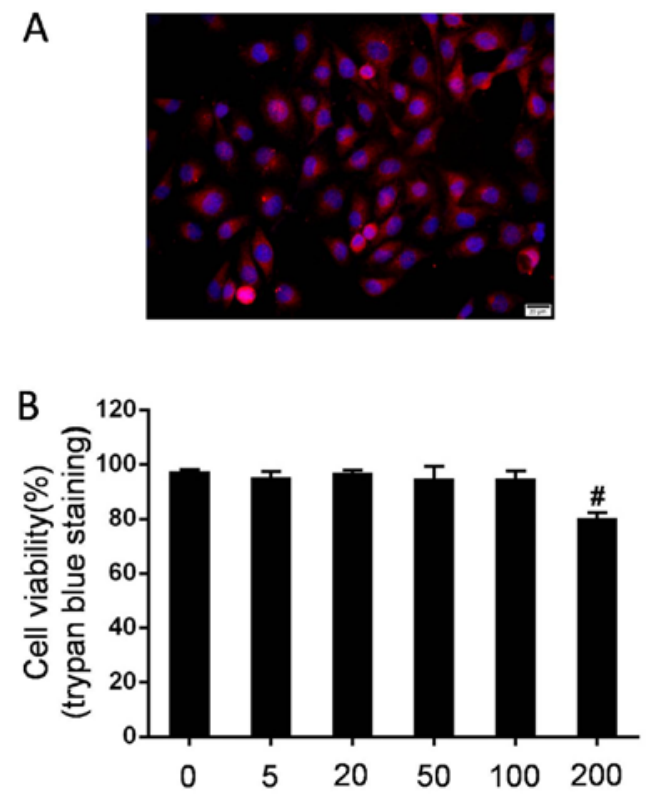

C
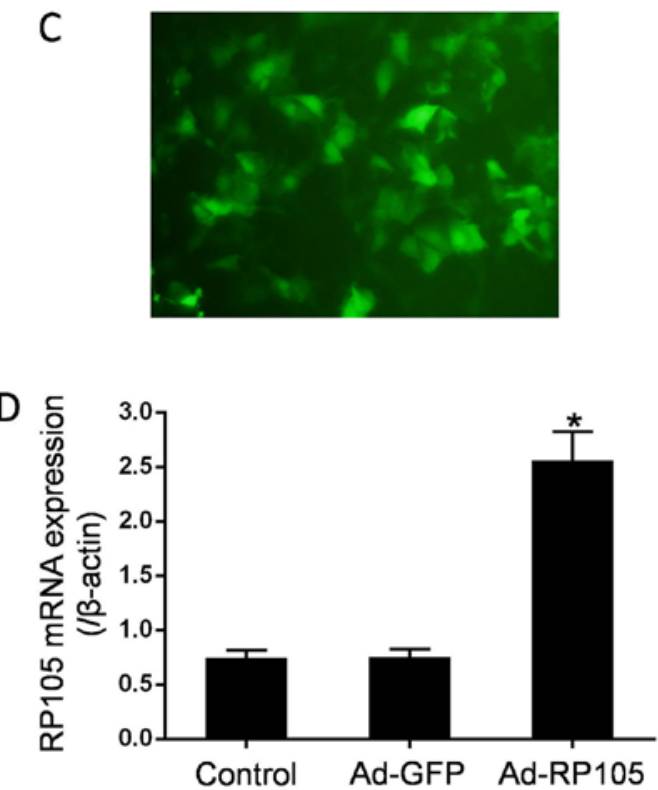

Figure 1. Efficiency of adenoviral transduction in cardiac microvascular endothelial cells (CMECs). (A) CD31 immunofluorescence staining was performed to identify the successful culture of CMECs. (B) Impact of viral transduction (MOI, 0-200) on CMEC viability measured by trypan blue staining. At 48 h after transduction of adenoviral vectors encoding RP105 (Ad-RP105) or Ad-GFP (MOI=100), (C) fluorescent microscopy and (D) reverse transription-quantitative polymerase chain reaction assay were performed to evaluate the success of adenoviral transfection, and to determine the efficient expression of RP105 mRNA. Scale bar, $20 \mu \mathrm{m}$. Data are presented as mean \pm standard deviation. ${ }^{\sharp} \mathrm{P}<0.05$ vs. group at $0 \mathrm{MOI}$; $\mathrm{P}<0.05$ vs. control group and Ad-GFP group. MOI, multiplicity of infection; GFP, green fluorescent protein.

size (Millipore, Billerica, MA, USA), as described by Wang et al (8). After H/R injury, CMECs were suspended with DMEM supplemented with 5\% FCS and added into the upper chamber. The lower chamber was filled with DMEM containing $20 \%$ FCS. At $12 \mathrm{~h}$ after incubation $\left(5 \% \mathrm{CO}_{2}\right.$ incubator at $\left.37^{\circ} \mathrm{C}\right)$, the migrated cells were fixed by $4 \%$ paraformaldehyde for $30 \mathrm{~min}$, and subsequently stained with $0.1 \%$ crystal violet. Migration was quantitatively detected through counting cells in 5 random microscopic fields (magnification, $\mathrm{x} 200$ ).

Apoptosis analysis. Apoptosis of CMECs was determined by Annexin V-APC/7-aminoactinomycin D (AV-APC/7-AAD) dual staining, based on the manufacturer's recommendations (26). CMECs were collected after H/R and stained with 7-AAD for $10 \mathrm{~min}$ in the dark at room temperature, followed by more staining with AV-APC. The AV-APC-positive and 7-AAD-negative cells were considered to be apoptotic CMECs.

Evaluation of IL-6 and tumor necrosis factor (TNF)- $\alpha$ secretion. The supernatants of cultured CMECs were collected for ELISA (12). IL-6 and TNF- $\alpha$ were measured by commercial ELISA kits in accordance with the manufacturer's instructions (R\&D Systems, Minneapolis, MN, USA).

Reverse transcription quantitative polymerase chain reaction (RT-qPCR) assay. Total RNA from CMECs was extracted by TRIzol reagent (Invitrogen; Thermo Fisher Scientific, Carlsbad, CA, USA), and reverse-transcribed into cDNA using a commercial synthesis kit (Thermo Fisher Scientific, Inc., Waltham, MA, USA) based on the manufacturer's protocol (25). RT-qPCR was performed using SYBR-Green (Fermentas, Waltham, MA, USA) with the ABI Prism 7500 system. PCR was performed as follows :50 ${ }^{\circ} \mathrm{C}$ for $2 \mathrm{~min}, 95^{\circ} \mathrm{C}$ for $10 \mathrm{~min}$, and 40 cycles of $95^{\circ} \mathrm{C}$ for $30 \mathrm{sec}$ and $60^{\circ} \mathrm{C}$ for $30 \mathrm{sec}$. Data were corrected to $\beta$-actin gene expression. The primers used for amplification were as follows: RP105, forward 5'-TGGGGACATTTGAGG ACATT-3'; and reverse 5'-GCTGTTAGGTCCAGCTCCTG-3'.

Western blot analysis. Western blotting was performed as previously described (25). The nuclear and cytoplasmic proteins were extracted in accordance with the manufacturer's protocol. Denatured proteins were separated by electrophoresis on $10 \%$ sodium dodecyl sulfate-polyacrylamide gel electrophoresis gels and transferred onto polyvinylidene fluoride membranes. Immunoblotting was probed by primary antibodies against glyceraldehyde 3-phosphate dehydrogenase (GAPDH; (1:1,000 dilution; sc-48166; Santa Cruz Biotechnology, Inc., Santa Cruz, CA, USA), lamin B (1:1,000 dilution; sc-20682; Santa Cruz Biotechnology, Inc.), RP105 (1:800 dilution; sc-27841; Santa Cruz Biotechnology, Inc.), TLR4 (1:500 dilution; sc-10741; Santa Cruz Biotechnology, Inc.), p38 MAPK (1:500 dilution; sc-535; Santa Cruz Biotechnology, Inc.), p-p38 MAPK (1:500 dilution; sc-7973; Santa Cruz Biotechnology, Inc.), JNK (1:500 dilution; sc-137020; Santa Cruz Biotechnology, Inc.), p-JNK (1:600 dilution; sc-6254; Santa Cruz Biotechnology, Inc.), ERK1/2 (1:300 dilution; sc-374239; Santa Cruz Biotechnology, Inc.), p-ERK1/2 (1:400 dilution; sc-7976; Santa Cruz Biotechnology, Inc.),

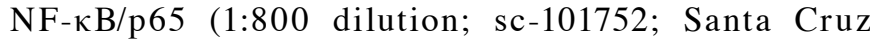
Biotechnology, Inc.), ICAM-1 (1:400 dilution; sc-31724; Santa Cruz Biotechnology, Inc.), IL-6 (1:800 dilution; sc-57315; Santa Cruz Biotechnology, Inc.) and TNF- $\alpha$ (1:600 dilution; sc-8301; Santa Cruz Biotechnology, Inc.). Protein bands were identified by horseradish peroxidase-conjugated rabbit antirat IgG secondary antibodies (1:800 dilution; bs-0346R-HRP; BIOSS, Boston, MA, USA). The outcomes were then visual- 
ized and analyzed by an enhanced chemiluminescence reagent kit (Thermo Fisher Scientific, Inc.). GAPDH and lamin B were used as internal loading controls for cytoplasmic proteins and nuclear NF- $\mathrm{kB} / \mathrm{p} 65$, respectively.

Statistical analysis. The statistical data are presented as mean \pm standard deviation. Comparisons between groups were performed with one-way analysis of variance and the Student-Newman-Keuls (SNK)-q test. P-values $<0.05$ were considered to indicate statistically significant differences.

\section{Results}

Efficiency of adenoviral transduction into CMECs. The confluence of the CMEC monolayer exhibited a cobblestone appearance under an inverted microscope. The typical CD31 immunofluorescence staining for CMECs identification was demonstrated (red, CD31; blue, nuclei dyed by DAPI) (Fig. 1A). At $48 \mathrm{~h}$ after the viruses were transduced at different MOI, the cytotoxic adenoviral effects were measured using $4 \%$ trypan blue dye. As shown in Fig. 1B, the adenovirus exerted no effect on CMEC viability at various MOI up to 100 , but exhibited a $80.3 \%$ decrease with an MOI of 200 . Thus, the MOI of 100 was utilized for the following experiments. Fluorescent microscopy and RT-qPCR analysis were performed to evaluate the success of the adenoviral transfection. Emission green fluorescence was highly displayed in CMECs transduced with Ad-GFP (Fig. 1C). Moreover, CMEC transduction with Ad-RP105 markedly promoted the expression of RP105 mRNA compared with that in control cells and Ad-GFP-treated cells $(\mathrm{P}<0.05)$ (Fig. 1D). These observations indicated the effectiveness of adenoviral transfection.

RP105 is minimally expressed in rat CMECs in response to $H / R$. As shown in Fig. 2, RP105 was highly expressed in rat CMECs under normoxic conditions, whereas the expression of RP105 at the protein level was considerably lower in response to $\mathrm{H} / \mathrm{R}$ ( $\mathrm{P}<0.05$ vs. control). Of note, compared with a coincident decrease in Ad-GFP-pretreated CMECs after H/R, a marked upregulation of RP105 was observed in Ad-RP105-transduced CMECs post- $\mathrm{H} / \mathrm{R}(\mathrm{P}<0.05)$. These results indicated the potential role of RP105 in the pathogenesis of CMEC-mediated $\mathrm{H} / \mathrm{R}$ injury.

RP105 enhances proliferation and migration of $H / R$-treated CMECs. Impairments in cell proliferation and migration ability are widely considered as important indices of CMEC dysfunction caused by $\mathrm{H} / \mathrm{R}$ injury $(4,8,10)$. To evaluate the effects of RP105 on CMEC malfunction, CCK-8 and Transwell chamber assays were performed after $\mathrm{H} / \mathrm{R}$ injury. As seen in Fig. 3A, cell proliferation in the $\mathrm{H} / \mathrm{R}$ group was markedly alleviated compared with the control group $(\mathrm{P}<0.05)$. However, pretreatment with Ad-RP105 in CMECs strongly reversed this decrease post-H/R ( $\mathrm{P}<0.05$ vs. $\mathrm{H} / \mathrm{R}$ group). In line with the results on proliferation, CMECs under $\mathrm{H} / \mathrm{R}$ conditions exhibited impaired migration ability as evidenced by decreased movement to the bottom surface of the Transwell chamber compared with control cells (Fig. 3B). Overexpression of RP105 contributed to the significantly enhanced migration of CMECs after $\mathrm{H} / \mathrm{R}(\mathrm{P}<0.05$ vs. $\mathrm{H} / \mathrm{R}$ group). Moreover, prolifer-

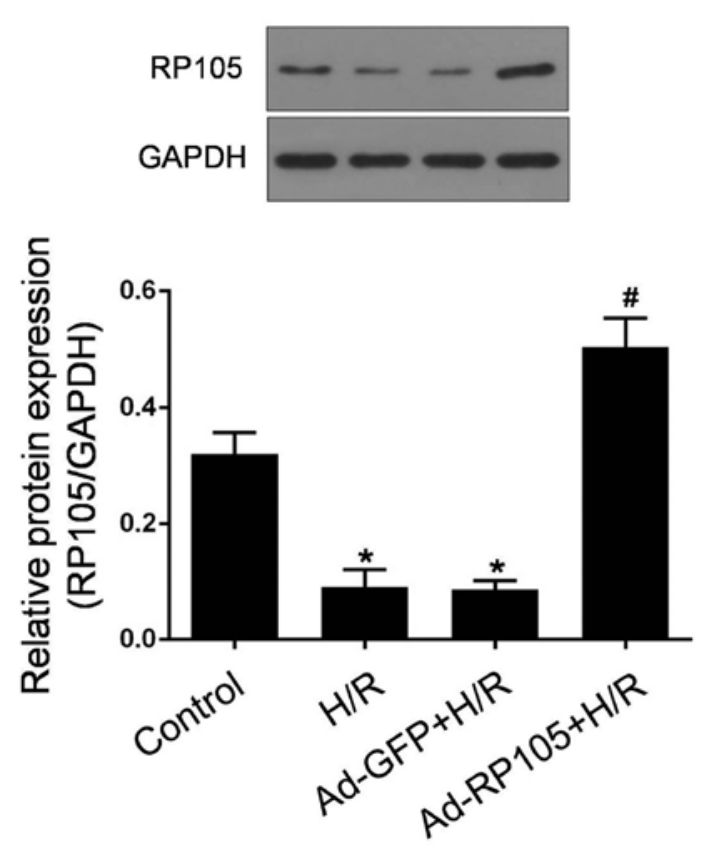

Figure 2. Radioprotective $105 \mathrm{kDa}$ protein (RP105) is minimally expressed in rat cardiac microvascular endothelial cells (CMECs) in response to hypoxia/reoxygenation (H/R). Representative western blot analyses and corresponding densitometry assays of RP105 protein are presented. Values are expressed as the mean \pm standard deviation of three independent experiments. ${ }^{*} \mathrm{P}<0.05$ vs. control group; ${ }^{\#} \mathrm{P}<0.05$ vs. $\mathrm{H} / \mathrm{R}$ or $\mathrm{Ad}-\mathrm{GFP}+\mathrm{H} / \mathrm{R}$ group. GFP, green fluorescent protein.

ation and migration were not affected by Ad-GFP transfection in $\mathrm{H} / \mathrm{R}$ CMECs ( $>0.05$ vs. $\mathrm{H} / \mathrm{R}$ group). These results provide evidence that RP105 markedly ameliorates the dysfunction of CMECs induced by $\mathrm{H} / \mathrm{R}$ injury.

RP105 attenuates H/R-related CMEC injury. Inflammation and apoptosis are considered as reliable indicators and crucial mechanisms for H/R-induced CMEC injury $(4,8,10)$. To further investigate the involvement of RP105 in the protection of CMECs against H/R injury, its effects on these parameters were evaluated. As regards the inflammatory response, a markedly higher secretion of IL- 6 and TNF- $\alpha$ was triggered after $\mathrm{H} / \mathrm{R}$, consistent with significantly increased expression levels of IL-6, TNF- $\alpha$ and ICAM-1 proteins in CMECs exposed to $\mathrm{H} / \mathrm{R}(\mathrm{P}<0.05$ vs. control; Fig. $4 \mathrm{~A}$ and $\mathrm{B})$. The transduction of Ad-RP105 resulted in a significant reduction of the abovementioned pro-inflammatory mediators compared with CMECs treated with $\mathrm{H} / \mathrm{R}(\mathrm{P}<0.05)$. In addition, similar patterns were observed in H/R-related activation of apoptosis (Fig. 4C). Using flow cytometric analysis, the number of apoptotic CMECs was found to be markedly increased following $H / R$ injury compared with control cells $(\mathrm{P}<0.05)$. However, overexpression of RP105 by Ad-RP105 led to a significant decrease of the apoptotic rate $(\mathrm{P}<0.05$ vs. $\mathrm{H} / \mathrm{R}$ group). Transduction with Ad-GFP in H/R CMECs exerted no obvious effect on the abovementioned injury parameters ( $\mathrm{P}>0.05 \mathrm{vs.} \mathrm{H/R}$ group). These findings indicate that RP105 possesses pleiotropic CMEC-protective effects against $H / R$ injury.

RP105 represses the expression of TLR4/MAPKs/NF- $\kappa B$ after $H / R$ injury. TLR4, MAPKs and NF- $\kappa \mathrm{B}$ have been 

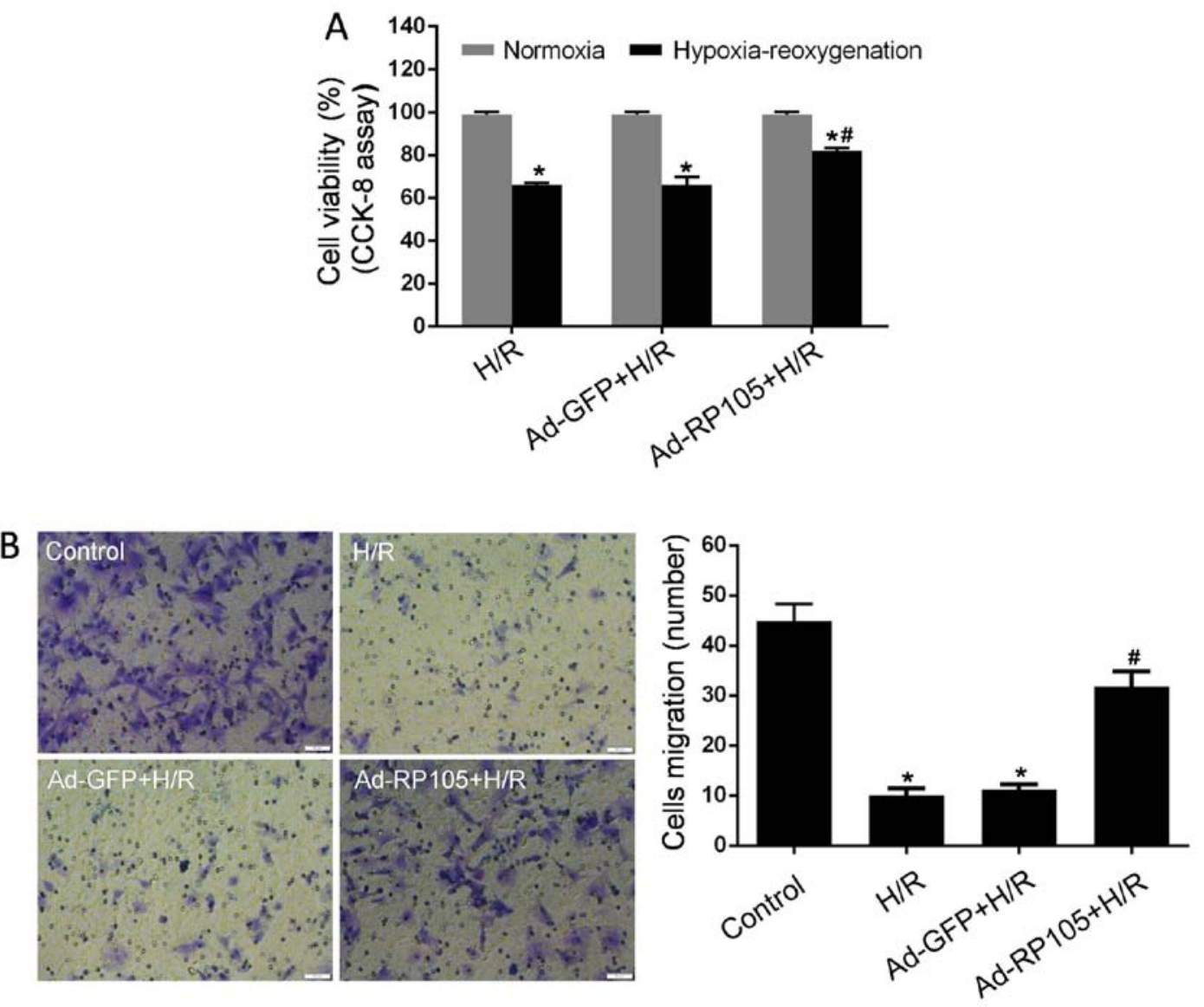

Figure 3. Radioprotective $105 \mathrm{kDa}$ protein (RP105) enhanced proliferation and migration of hypoxia/reoxygenation (H/R)-treated cardiac microvascular endothelial cells (CMECs). After H/R treatment, cell proliferation and migration abilities were examined by (A) cell counting kit-8 (CCK-8) and (B) Transwell chamber analysis. The results are expressed as means \pm standard deviation of three separate experiments. Scale bar, $25 \mu \mathrm{m}$. ${ }^{~} \mathrm{P}<0.05$ vs. control group; ${ }^{\#} \mathrm{P}<0.05$ vs. H/R or Ad-GFP+H/R group. GFP, green fluorescent protein.

demonstrated to participate, in combination and/or separately, in the pathological process of microvascular endothelial cell injury under ischemic conditions $(10,27)$. TLR4 acts as a common upstream sensor and stimulates MAPKs/NF- $\kappa \mathrm{B}$ signaling, which may constitute the original mechanism underlying H/R-related CMEC injury. To detect whether RP105 regulates the activities of MAPKs and $\mathrm{NF}-\kappa \mathrm{B}$ through antagonizing TLR4 in CMECs post-H/R, western blotting was used to measure the expressions of TLR4, p38 MAPK, p-p38 MAPK, JNK, p-JNK, ERK1/2,

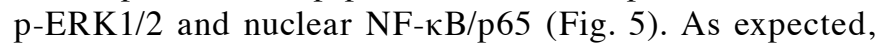
there was a markedly increased expression of TLR4, p-p38 MAPK, p-JNK, p-ERK1/2 and nuclear NF- $\kappa$ B/p65 proteins in CMECs following $\mathrm{H} / \mathrm{R}$ injury compared with control cells $(\mathrm{P}<0.05)$. However, $\mathrm{H} / \mathrm{R}$-induced activation of TLR4/MAPKs/NF- $\kappa$ B signaling was significantly abolished by Ad-RP105 pretreatment in CMECs (vs. H/R, P<0.05). Moreover, the levels of total p38 MAPK, ERK1/2 and JNK exhibited no obvious differences among the four groups $(\mathrm{P}>0.05)$. Ad-GFP transduction exerted no significant effect on the expression of those proteins (vs. H/R group, $\mathrm{P}>0.05$ ). Taken together, these findings indicate that the protective effects of RP105 may be closely correlated with the blockade of TLR4/MAPKs/NF- $\mathrm{B}$-mediated H/R injury in CMECs.

\section{Discussion}

Although the cardiomyocyte-protective role of RP105 has been previously demonstrated, its involvement and causal properties in ameliorating H/R injury of coronary CMECs remain unclear. To the best of our knowledge, the present study is the first to elucidate the effects of RP105 in CMEC H/R injury. The results indicated that RP105 is minimally expressed in H/R-treated CMECs. Overexpression of RP105 via adenoviral vectors significantly contributed to a reduction of IL-6, TNF- $\alpha$ and ICAM-1 expression, as well as fewer apoptotic CMECs relative to those in un-transduced and Ad-GFP-transduced CMECs subjected to H/R injury. Moreover, the proliferation and migration of $\mathrm{H} / \mathrm{R}$-treated CMECs were also strongly promoted by Ad-RP105 transduction. Mechanistic studies reported that RP105 overexpression blunts the expression of TLR4, p-ERK1/2, p-p38 MAPK, p-JNK and nuclear $\mathrm{NF}-\kappa \mathrm{B} / \mathrm{p} 65$. Therefore, RP105 may be used as a therapeutic approach against $\mathrm{CMEC} \mathrm{H} / \mathrm{R}$ injury.

Inflammatory responses have been widely investigated due to their impact on the initiation and expansion of myocardial I/R injury (12). Damaging inflammatory cells, including neutrophils, macrophages and monocytes, are recruited to the ischemic regions, and the concomitant cytotoxic outcomes 

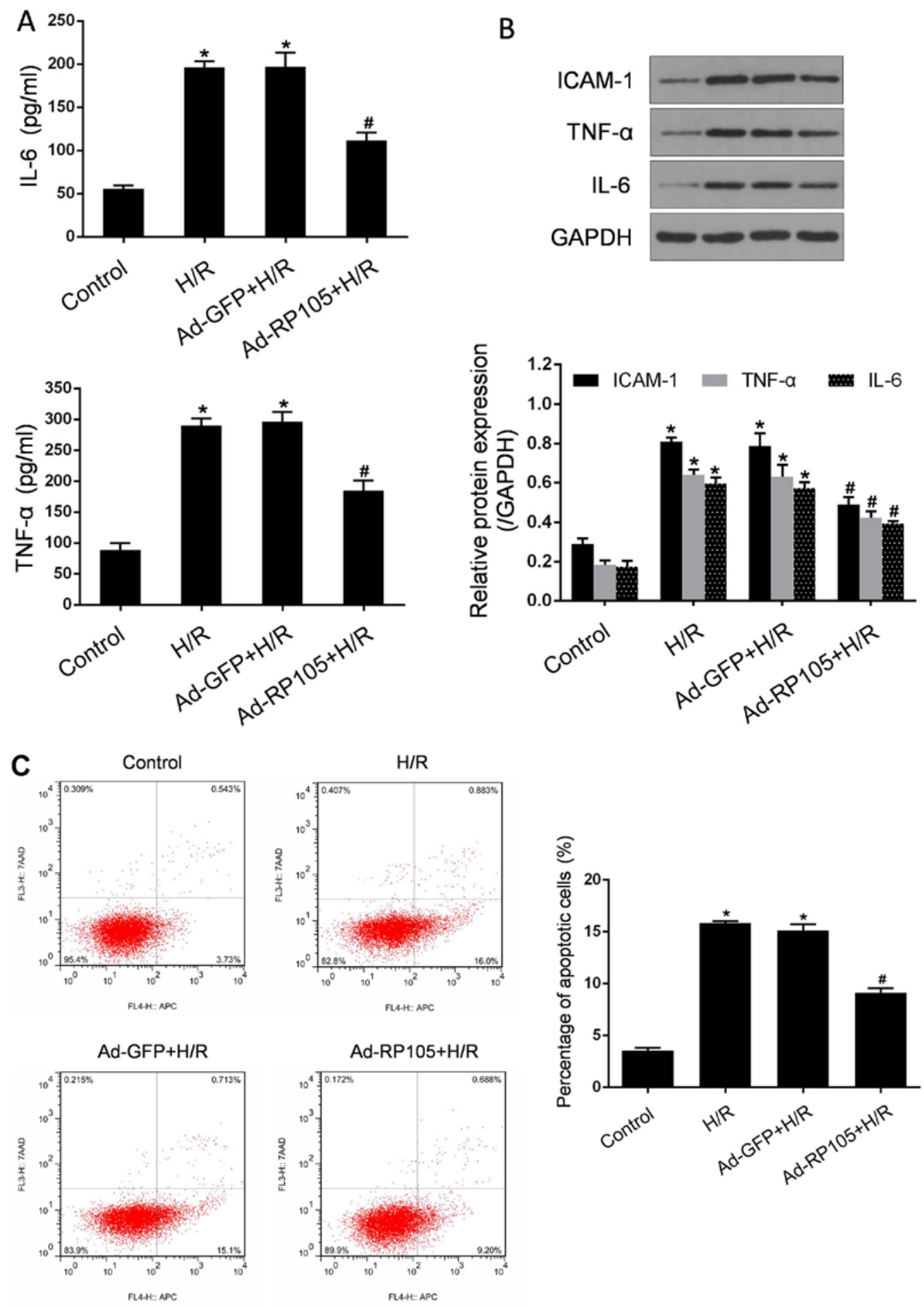

Figure 4. Radioprotective $105 \mathrm{kDa}$ protein (RP105) attenuated hypoxia/reoxygenation (H/R)-related cardiac microvascular endothelial cell (CMEC) injury. (A) The release of interleukin (IL)- 6 and tumor necrosis factor (TNF)- $\alpha$ were detected by ELISA in the culture supernatant. (B) Representative western blot analyses of IL-6, TNF- $\alpha$ and intercellular adhesion melecule-1 (ICAM-1) and corresponding densitometry analysis. (C) Original representative dot-plot diagrams and percentage of apoptotic CMECs on Annexin V-APC/7-aminoactinomycin D (AV-APC/7-ADD) flow cytometry. The results are expressed as means \pm standard deviation in three biological replicates, ${ }^{*} \mathrm{P}<0.05$ vs. control group; ${ }^{\#} \mathrm{P}<0.05$ vs. $\mathrm{H} / \mathrm{R}$ or Ad-GFP+H/R group. GFP, green fluorescent protein.

comprise the central mechanisms causing acute losses and delayed remodeling of cardiomyocytes and adjacent vascular endothelial cells in case of $\mathrm{I} / \mathrm{R}(1,4)$. CMECs, in particular, act as central mediators in detecting damage-associated molecular patterns and determine cardiac responses to I/R injury (8), among which the activation of TLR4 and the upregulation of ICAM-1 synergistically induce the recruitment of inflammatory cells $(2,12)$. Inflammatory stimulation leads to the upregulation of ICAM-1, an increase in cytokine production, as well as the activation of apoptosis $(12,28)$. In addition, recent studies identified a potential correlation between TLR4 inhibition and repressed synthesis of ICAM-1 in endothelial cells exposed to high glucose stimulation, and TLR4 is likely to be a key modulator in the regulation of ICAM-1 expression 

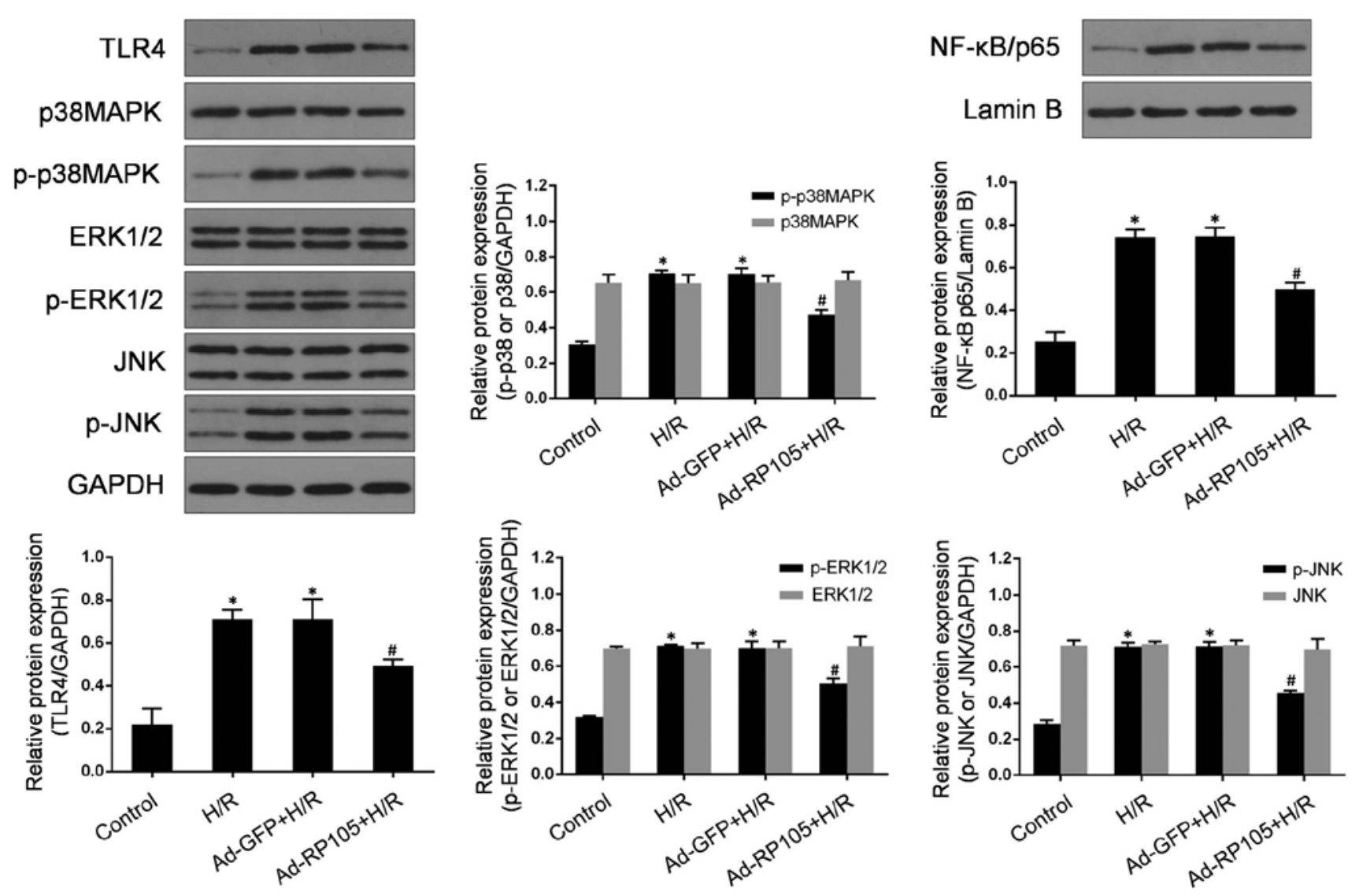

Figure 5. Radioprotective $105 \mathrm{kDa}$ protein (RP105) repressed the expression of Toll-like receptor 4 (TLR4)/MAPKs/NF- $\mathrm{BB}$ in hypoxia/reoxygenation (H/R) injury. Original representative western blot analyses and relative protein expression of TLR4, p38 MAPK, p-p38 MAPK, extracellular signal-regulated kinase 1/2 (ERK1/2), p-ERK1/2, c-Jun N-terminal kinase (JNK) and p-JNK, with unclear nuclear factor (NF)- $\kappa B / p 65$. Values are presented as mean \pm standard deviation of three separate experiments. ${ }^{*} \mathrm{P}<0.05$ vs. control group; ${ }^{\text {}} \mathrm{P}<0.05 \mathrm{vs}$. H/R or Ad-GFP+H/R group. GFP, green fluorescent protein; MAPK, mitogen-activated protein kinase.

under inflammatory conditions $(29,30)$. The present study demonstrated a previously unknown CMEC-protective role of RP105 against $\mathrm{H} / \mathrm{R}$ injury by suppressing the expression and secretion of pro-inflammatory cytokines (IL-6 and TNF- $\alpha$ ), and by limiting ICAM-1 expression, which are paralleled by TLR4 downregulation. In terms of the importance of ICAM-1 and TLR4 in initiating and amplifying inflammatory responses in CMECs, RP105 may play a key role in repressing $H / R$ injury.

Previous studies have demonstrated that the activation of TLR4/NF- $\kappa B$ signaling has a detrimental effect in the dysfunction of CMECs in response to H/R (10). However, the exact mechanisms that sensitize CMECs to H/R injury remain incompletely understood and the endogenous mechanisms that may regulate the disassociation between TLR4 and CMEC H/R injury are not known. Previous studies revealed that the activity of p-ERK1/2 was involved in the inflammatory injury of brain microvascular endothelial cells caused by mock ischemic treatment (31), and its participation in inducing apoptosis under hypoxic conditions was also verified $(32,33)$. Moreover, the pro-inflammatory and -apoptotic patterns of p-p38 MAPK and p-JNK activation in the ischemic endothelium have also been extensively investigated (10-15,27,34). Upon stimulation in the endothelium, TLR4 triggers the inflammation and apoptosis cascades, partly through phosphorylation of MAPKs and NF- $\mathrm{BB}$ activation, all of which may be abolished by TLR4 and/or MAPKs inhibition (10,12-15,27,33). However, whether MAPKs and NF- $\kappa B$ participate in the multifarious pathogenesis of CMEC H/R injury via TLR4-mediated mechanisms has not been fully elucidated. Our study demonstrated that TLR4/MAPKs/NF-кB signaling was significantly upregulated in CMECs subjected to H/R injury, followed by promotion of inflammation and activation of apoptosis. Therefore, we hypothesized that RP105, known for its TLR4-inhibitory properties, was able to abolish the causal association between TLR4/MAPKs/NF- $\kappa B$ signaling and CMEC H/R injury. Our findings, therefore, indicate that RP105, particularly its CMEC-protective properties, may provide a promising therapeutic approach to ameliorating $\mathrm{H} / \mathrm{R}$ injury.

Of note, the role of RP105 in cardiovascular disease has been extensively investigated. A recent study reported the protective properties of RP105 in vascular remodeling and myocardial infarction via TLR4-inhibitory mechanisms $(21,22)$. Our previous findings also demonstrated the cardiomyocyte-protective properties of RP105 against I/R injury through downregulation of TLR4 (11). Although RP105 acted as a structural and functional inhibitor of TLR4, its role may not be merely confined to TLR4-dependent approaches (11). For example, researchers observed facilitated vein graft lesions in $\mathrm{RP} 105^{-/}$mice resulting from the enhancement of chemoattractant chemokine ligand-2 production and inflammatory response (20). RP105 deficiency alleviated 
atherosclerotic plaque development through downregulation of C-C chemokine receptor-2 expression (35). In addition, RP105 plays a major regulatory role in adipose tissue inflammation independently of TLR4-mediated pathways (36). It is becoming increasingly clear that the biological actions of RP105 are mediated through TLR4-independent pathways, and one mechanism is possibly associated with the RP105/PI3K/Akt axis (11). The findings of the present study established that RP105 attenuated CMEC H/R injury in a TLR4-dependent manner. Whether other causal mechanisms are involved and RP105 modulation may provide pleiotropic protective effects requires further elucidation.

The protective properties of RP105 in the setting of $I / R$ injury were primarily ascribed to be targeted on cardiomyocytes $(11,18)$. In our previous studies, regional transduction of Ad-RP105 significantly limited cardiomyocyte-related inflammatory, apoptotic and autophagic responses, along with limiting infarct size and attenuation of cardiac function in $\mathrm{I} / \mathrm{R}$ injury $(11,18)$. However, the contribution of RP105 to CMEC H/R injury remains largely unknown. Recently, Karper et al demonstrated that RP105 was implicated in the process of VSMC-mediated neointima formation and post-interventional vascular remodeling (21). RP105 deficiency may disturb migration of monocytes in atherosclerotic plaque formation (35). These observations suggest that RP105 may play important roles in cardiovascular pathological conditions that are not limited to cardiomyocytes. In the present study, it was demonstrated that RP105 ameliorated CMEC inflammation and apoptosis caused by H/R. The effects of RP105 on cell proliferation and migration were also expounded for the first time. Our findings identified CMECs as the target of the microvascular endothelium-protective actions of RP105, as well as expanded its beneficial effects beyond I/R-treated cardiomyocytes. Interestingly, previous studies revealed the dual role of RP105 in regulating TLR4 and TLR2, with RP105 inhibiting the activity of TLR4 and its downstream signaling molecules and, conversely, facilitating TLR2-induced inflammatory response (17). The detrimental effects of TLR2 activation on coronary vascular endothelial cells during $\mathrm{I} / \mathrm{R}$ has been previously investigated (37). However, whether RP105 regulates CMEC-associated $\mathrm{H} / \mathrm{R}$ injury through a TLR2-dependent mechanism requires further confirmation.

Taken together, the findings presented herein highlight the importance of RP105 and its role in CMEC protection from $\mathrm{H} / \mathrm{R}$ injury. The underlying mechanism at least partly involves the inactivation of TLR4/MAPKs/NF- $\mathrm{KB}$ signaling. Therefore, interventional strategies that selectively regulate RP105 may be potential candidates for the mitigation of H/R injury. Future investigations are required, focusing on endogenous targets, such as specific microRNAs, IncRNAs and/or circRNAs in CMECs, by means of alternatively regulating RP105 followed by TLR4 modulation, which may represent a novel modality for protecting cells against I/R injury.

\section{Acknowledgements}

The present study was supported by the National Natural Science Foundation of China (grant nos. 81200156, 81200088, 81470387 and 81570331) and the Fundamental Research Funds for the Central Universities (grant no. 20120141120079).

\section{Competing interests}

The authors declare that they have no competing interests.

\section{References}

1. Ibanez B, Heusch G, Ovize M and Van de Werf F: Evolving therapies for myocardial ischemia/reperfusion injury. J Am Coll Cardiol 65: 1454-1471, 2015.

2. Zhang Y, Zhou H, Wu W, Shi C, Hu S, Yin T, Ma Q, Han T, Zhang Y, Tian F, et al: Liraglutide protects cardiac microvascular endothelial cells against hypoxia/reoxygenation injury through the suppression of the SR-Ca(2+)-XO-ROS axis via activation of the GLP-1R/PI3K/Akt/survivin pathways. Free Radic Biol Med 95: 278-292, 2016.

3. Cui H,Li X, Li N, Qi K, Li Q, Jin C, Zhang Q, Jiang L and Yang Y: Induction of autophagy by Tongxinluo through the MEK/ERK pathway protects human cardiac microvascular endothelial cells from hypoxia/reoxygenation injury. J Cardiovasc Pharmacol 64: 180-190, 2014.

4. Liu Y, Lian K, Zhang L, Wang R, Yi F, Gao C, Xin C, Zhu D, Li Y, Yan W, et al: TXNIP mediates NLRP3 inflammasome activation in cardiac microvascular endothelial cells as a novel mechanism in myocardial ischemia/reperfusion injury. Basic Res Cardiol 109: 415, 2014.

5. Li JM, Mullen AM and Shah AM: Phenotypic properties and characteristics of superoxide production by mouse coronary microvascular endothelial cells. J Mol Cell Cardiol 33: 1119-1131, 2001.

6. Zhou Y, Zhang Y, Gao F, Guo F, Wang J, Cai W, Chen Y, Zheng J and Shi G: N-n-butyl haloperidol iodide protects cardiac microvascular endothelial cells from hypoxia/reoxygenation injury by down-regulating Egr-1 expression. Cell Physiol Biochem 26: 839-848, 2010.

7. Qi XF,Li YJ,Chen ZY, Kim SK, Lee KJ and Cai DQ: Involvement of the FoxO3a pathway in the ischemia/reperfusion injury of cardiac microvascular endothelial cells. Exp Mol Pathol 95: 242-247, 2013.

8. Wang J, Hong Z, Zeng C, Yu Q and Wang H: NADPH oxidase 4 promotes cardiac microvascular angiogenesis after hypoxia/reoxygenation in vitro. Free Radic Biol Med 69: 278-288, 2014.

9. Coulombe KL, Bajpai VK, Andreadis ST and Murry CE: Heart regeneration with engineered myocardial tissue. Annu Rev Biomed Eng 16: 1-28, 2014.

10. Zhang Z,Li W, Sun D, Zhao L, Zhang R, Wang Y,Zhou X, Wang H and Cao F: Toll-like receptor 4 signaling in dysfunction of cardiac microvascular endothelial cells under hypoxia/reoxygenation. Inflamm Res 60: 37-45, 2011.

11. Guo X, Jiang $\mathrm{H}$ and Chen J: RP105-PI3K-Akt axis: A potential therapeutic approach for ameliorating myocardial ischemia/reperfusion injury. Int J Cardiol 206: 95-96, 2016.

12. Hinkel R, Lange P, Petersen B, Gottlieb E, Ng JK, Finger S, Horstkotte J, Lee S, Thormann M, Knorr M, et al: Heme oxygenase-1 gene therapy provides cardioprotection via control of post-ischemic inflammation: An experimental study in a preclinical pig model. J Am Coll Cardiol 66: 154-165, 2015.

13. Zanotti G, Casiraghi M, Abano JB, Tatreau JR, Sevala M, Berlin H, Smyth S, Funkhouser WK, Burridge K, Randell SH, et al: Novel critical role of Toll-like receptor 4 in lung ischemia-reperfusion injury and edema. Am J Physiol Lung Cell Mol Physiol 297: L52-L63, 2009.

14. Chen Y, Huang XJ, Yu N, Xie Y, Zhang K, Wen F, Liu H and Di Q: HMGB1 contributes to the expression of P-Glycoprotein in mouse epileptic brain through Toll-Like receptor 4 and receptor for advanced glycation end products. PLoS One 10: e0140918, 2015.

15. Mountain DJ, Singh M and Singh K: Downregulation of VEGF-D expression by interleukin-1beta in cardiac microvascular endothelial cells is mediated by MAPKs and PKCalpha/beta1. J Cell Physiol 215: 337-343, 2008.

16. Liu RR, Li J, Gong JY, Kuang F, Liu JY, Zhang YS, Ma QL, Song CJ, Truax AD, Gao F, et al: MicroRNA-141 regulates the expression level of ICAM-1 on endothelium to decrease myocardial ischemia-reperfusion injury. Am J Physiol Heart Circ Physiol 309: H1303-H1313, 2015.

17. Liu B, Zhang N, Liu Z, Fu Y, Feng S, Wang S, Cao Y, Li D, Liang D, $\mathrm{Li} \mathrm{F}$, et al: RP105 involved in activation of mouse macrophages via TLR2 and TLR4 signaling. Mol Cell Biochem 378: 183-193, 2013. 
18. Li X, Yang J, Yang J, Dong W, Li S, Wu H and Li L: RP105 protects against myocardial ischemia-reperfusion injury via suppressing TLR4 signaling pathways in rat model. Exp Mol Pathol 100: 281-286, 2016.

19. Hijiya N, Miyake K, Akashi S, Matsuura K, Higuchi Y and Yamamoto S: Possible involvement of toll-like receptor 4 in endothelial cell activation of larger vessels in response to lipopolysaccharide. Pathobiology 70: 18-25, 2002.

20. Wezel A, de Vries MR, Maassen JM, Kip P, Peters EA, Karper JC, Kuiper J, Bot I and Quax PH: Deficiency of the TLR4 analogue RP105 aggravates vein graft disease by inducing a pro-inflammatory response. Sci Rep 6: 24248, 2016

21. Karper JC, Ewing MM, de Vries MR, de Jager SC, Peters EA, de Boer HC, van Zonneveld AJ, Kuiper J, Huizinga EG, Brondijk TH, et al: TLR accessory molecule RP105 (CD180) is involved in post-interventional vascular remodeling and soluble RP105 modulates neointima formation. PLoS One 8: e67923, 2013.

22. Louwe MC, Karper JC, de Vries MR, Nossent AY, Bastiaansen AJ, van der Hoorn JW, Willems van Dijk K, Rensen PC, Steendijk P, Smit JW, et al: RP105 deficiency aggravates cardiac dysfunction after myocardial infarction in mice. Int J Cardiol 176: 788-793, 2014.

23. Xia JB, Liu GH, Chen ZY, Mao CZ, Zhou DC, Wu HY, Park KS, Zhao H, Kim SK, Cai DQ, et al: Hypoxia/ischemia promotes CXCL10 expression in cardiac microvascular endothelial cells by NFkB activation. Cytokine 81: 63-70, 2016.

24. Yang J, Guo X, Yang J, Ding JW, Li S, Yang R, Fan ZX and Yang CJ: RP105 protects against apoptosis in ischemia/reperfusion-induced myocardial damage in rats by suppressing TLR4-mediated signaling pathways. Cell Physiol Biochem 36: 2137-2148, 2015.

25. Yang J, Chen L, Ding J,Zhang J, Fan Z, Yang C, Yu Q and Yang J: Cardioprotective effect of miRNA-22 on hypoxia/reoxygenation induced cardiomyocyte injury in neonatal rats. Gene 579: 17-22, 2016.

26. Choi HS, Kim MK, Choi YK, Shin YC, Cho SG and Ko SG: Rhus verniciflua Stokes (RVS) and butein induce apoptosis of paclitaxel-resistant SKOV-3/PAX ovarian cancer cells through inhibition of AKT phosphorylation. BMC Complement Altern Med 16: 122, 2016.

27. Ye EA and Steinle JJ: miR-146a attenuates inflammatory pathways mediated by $\mathrm{TLR} / \mathrm{NF}-\kappa \mathrm{B}$ and $\mathrm{TNF} \alpha$ to protect primary human retinal microvascular endothelial cells grown in high glucose. Mediators Inflamm 2016: 3958453, 2016.
28. Shi J, Zhou J and Zhang M: Microcystins induces vascular inflammation in human umbilical vein endothelial cells via activation of NF- $\kappa$ B. Mediators Inflamm 942159: 2015, 2015.

29. Mudaliar H, Pollock C, Ma J, Wu H, Chadban S and Panchapakesan U: The role of TLR2 and 4-mediated inflammatory pathways in endothelial cells exposed to high glucose. PLoS One 9: e108844, 2014.

30. Sawa Y, Ueki T, Hata M, Iwasawa K, Tsuruga E, Kojima H, Ishikawa $\mathrm{H}$ and Yoshida S: LPS-induced IL-6, IL-8, VCAM-1, and ICAM-1 expression in human lymphatic endothelium. J Histochem Cytochem 56: 97-109, 2008.

31. Li F, Li W, Li X, Li F, Zhang L, Wang B, Huang G, Guo X, Wan L, Liu $Y$, et al: Geniposide attenuates inflammatory response by suppressing P2Y14 receptor and downstream ERK1/2 signaling pathway in oxygen and glucose deprivation-induced brain microvascular endothelial cells. J Ethnopharmacol 185: 77-86, 2016.

32. Cheng F, Lan J, Xia W, Tu C, Chen B, Li S and Pan W: Folic acid attenuates vascular endothelial cell injury caused by hypoxia via the inhibition of ERK1/2/NOX4/ROS pathway. Cell Biochem Biophys 74: 205-211, 2016.

33. Li J,ZhangZ,LvL,QiaoH,ChenX andZou C: (-)-Epigallocatechin gallate inhibits asymmetric dimethylarginine-induced injury in human brain microvascular endothelial cells. Neurochem Res 41: 1868-1876, 2016.

34. Wang LW, Chang YC, Chen SJ, Tseng CH, Tu YF, Liao NS, Huang CC and Ho CJ: TNFR1-JNK signaling is the shared pathway of neuroinflammation and neurovascular damage after LPS-sensitized hypoxic-ischemic injury in the immature brain. J Neuroinflammation 11: 215, 2014.

35. Wezel A, van der Velden D, Maassen JM, Lagraauw HM, de Vries MR, Karper JC, Kuiper J, Bot I and Quax PH: RP105 deficiency attenuates early atherosclerosis via decreased monocyte influx in a CCR2 dependent manner. Atherosclerosis 238: 132-139, 2015.

36. Nagai Y, Watanabe $Y$ and Takatsu K: The TLR family protein RP105/MD-1 complex: A new player in obesity and adipose tissue inflammation. Adipocyte 2: 61-66, 2013

37. Jin C, Cleveland JC, Ao L, Li J, Zeng Q, Fullerton DA and Meng X: Human myocardium releases heat shock protein 27 (HSP27) after global ischemia: The proinflammatory effect of extracellular HSP27 through toll-like receptor (TLR)-2 and TLR4. Mol Med 20: 280-289, 2014. 